\title{
LPCAT1 overexpression promotes the progression of hepatocellular carcinoma
}

\author{
Rong-Quan He1, Jian-Di Li², Xiu-Fang Du², Yi-Wu Dang², Lin-Jie Yang ${ }^{2}$, Zhi-Guang Huang ${ }^{2}$, Li-Min Liư ${ }^{3}$, \\ Liu-Feng Liao ${ }^{4}$, Hong Yang ${ }^{5}$ and Gang Chen ${ }^{2^{*}}$ (D)
}

\begin{abstract}
Background: Hepatocellular carcinoma (HCC) remains one of the most common malignant neoplasms. Lysophosphatidylcholine acyltransferase 1 (LPCAT1) plays a key role in the lipid remodelling and is correlated with various neoplasms. Nonetheless, the biological functions and molecular mechanisms of LPCAT1 underlying HCC remain obscure.

Methods: In the present study, we investigated the role of LPCAT1 in the progression of HCC. In-house RT-qPCR, tissue microarrays, and immunohistochemistry were performed to detect the expression levels and the clinical value of LPCAT1 in HCC. External datasets were downloaded to confirm the results. Proliferation, migration, invasiveness, cell cycle, and apoptosis assays were conducted to reveal the biological effects LPCAT1 has on SMMC-7721 and Huh7 cells. HCC differentially expressed genes and LPCAT1 co-expressed genes were identified to explore the molecular mechanisms underlying HCC progression.
\end{abstract}

Results: LPCAT1 showed upregulated expression in 3715 HCC specimens as opposed to 3105 non-tumour specimens. Additionally, LPCAT1 might be an independent prognostic factor for HCC. LPCAT1-knockout hampered cellular proliferation, migration, and metastasis in SMMC-7721 and Huh7 cells. More importantly, the cell cycle and chemical carcinogenesis were the two most enriched signalling pathways.

Conclusions: The present study demonstrated that increased LPCAT1 correlated with poor prognosis in HCC patients and fuelled HCC progression by promoting cellular growth, migration, and metastasis.

Keywords: Hepatocellular carcinoma, Lysophosphatidylcholine acyltransferase 1, Functional experiment, Molecular mechanism, Clinical applications

\section{Background}

Hepatocellular carcinoma (HCC), characterized by high molecular heterogeneity, serves as the predominant subtype of liver cancer [1,2]. HCC is the third most frequent cause of cancer deaths worldwide, and ranks fifth and seventh in male and female malignancy [3], respectively. According to American Cancer Statistics 2021, there will be approximately 40,000 new cancer cases and 30,000

\footnotetext{
*Correspondence: chengang@gxmu.edu.cn

${ }^{2}$ Department of Pathology, First Affiliated Hospital of Guangxi Medical University, No. 6 Shuangyong Rd, Guangxi Zhuang Autonomous Region, Nanning 530021, People's Republic of China

Full list of author information is available at the end of the article
}

deaths per year, with a 5-year relative survival rate of only $20 \%$ [3]. HCC is a complex and multistage disease that may be explained by genetic and epigenetic changes [4-8]. Despite the development of surgery and combined treatment, as well as progress in early diagnosis and interventional therapy, most patients are still diagnosed in the advanced stage of $\mathrm{HCC}$ with a low 5-year survival rate and poor prognosis [9-11]. Therefore, clarifying the pathogenic mechanisms of HCC is essential for establishing novel potential therapeutic strategies and improving the prognosis of advanced stage HCC patients.

As a basic material of life, phospholipids play an important role in maintaining normal cellular structures, homeostasis, and fundamental cellular biological original author(s) and the source, provide a link to the Creative Commons licence, and indicate if changes were made. The images or other third party material in this article are included in the article's Creative Commons licence, unless indicated otherwise in a credit line to the material. If material is not included in the article's Creative Commons licence and your intended use is not permitted by statutory regulation or exceeds the permitted use, you will need to obtain permission directly from the copyright holder. To view a copy of this licence, visit http://creativecommons.org/licenses/by/4.0/. The Creative Commons Public Domain Dedication waiver (http://creativeco mmons.org/publicdomain/zero/1.0/) applies to the data made available in this article, unless otherwise stated in a credit line to the data. 
functions [12]. However, abnormal lipid metabolism disturbs cellular activities and drives cancer formation by affecting cell differentiation, proliferation, and apoptosis [13]. Lysophosphatidylcholine acyltransferase 1 (LPCAT1), encoded by LPCAT1, is a phospholipid biosynthesis/remodelling enzyme that plays a key role in the lipid remodelling pathway referred to as Lands' cycle [14, 15]. LPCAT1 participates in phospholipid metabolism by catalysing the conversion from lysophosphatidylcholine to phosphatidylcholine and has been demonstrated to be correlated with various neoplasms, including hepatoma, acute myeloid leukaemia [13], breast cancer [16], clear cell renal cell carcinoma [17], colorectal cancer [18], esophageal cancer, lung adenocarcinoma [19], oral squamous cell carcinoma [20], and prostate cancer [21]. Thus far, only one study, limited in sample size, demonstrates that LPCAT1 is upregulated in $37 \mathrm{HCC}$ tissue samples [22]. During the process of tumour initiation and progression, increased LPCAT1 promotes the synthesis and remodelling of lipids in the lipid-dependent membrane structures of rapidly proliferating cancer cells [16]. Additionally, LPCAT1 induces metastasis of cancer through complicated mechanisms, such as the lipid metabolism pathway and PI3K/ AKT/ MYC pathway [23]. Moreover, the alteration of the phospholipid component regulated by LPCAT1 promotes cell proliferation and membrane fluidity, thus causing the occurrence and development of clear cell renal cell carcinoma [17]. Collectively, such findings indicate the vital roles $L P C A T 1$ play in tumour onset and progression. Nonetheless, the exact biological functions and precise molecular mechanisms of LPCAT1 underlying $\mathrm{HCC}$ remain obscure. More experiments must be conducted to determine the key roles of LPCAT1 in $\mathrm{HCC}$ and to design novel therapeutic targets.

In this study, the authors aimed to verify the expression of LPCAT1 in large numbers of clinical HCC tissue samples and to explore the biological functions of LPCAT1 in HCC cells. Furthermore, the potential molecular mechanisms and therapeutics of LPCAT1 underlying HCC were probed.

\section{Methods}

\section{HCC-derived cell lines and tissue samples}

HCC-derived SMMC-7721 and Huh7 cell lines were obtained from the Cell Bank of the Chinese Academy of Sciences, Shanghai, People's Republic of China [24]. The cellular identity was confirmed by short tandem repeat profiles. All cells were cultured in Dulbecco's modified Eagle's medium (DMEM) containing 10\% fetal bovine serum (FBS) (Sigma), as formerly reported [25].

To detect the mRNA expression level of LPCAT1, 204 HCC tissue specimens and 204 non-tumour hepatic tissue specimens were obtained from the Affiliated Cancer
Hospital of Guangxi Medical University, Nanning, People's Republic of China. The clinicopathological parameters and follow-up information of each patient were recorded. Informed consent was provided. To determine the protein expression level of LPCAT1 in HCC, a total of four tissue microarrays, containing LVC1505, LVC1531, LVC1601, and LVC1602, were purchased from Fanpu Biotech, Inc. (http://www.fanpu.com), Guilin, People's Republic of China. A total of 315 HCC tissue specimens and 195 non-HCC tissue specimens were prepared to perform immunohistochemistry (IHC) staining. The inclusion criteria for patients in this study are as follows, (I) the pathological diagnosis should be primary HCC; (II) there should be sufficient tissue specimens for RNA extraction or IHC. To avoid confounding effect, HCC patient with intrahepatic cholangiocarcinoma was excluded in this study. The present research was authorised by the ethics committee of the First Affiliated Hospital of Guangxi Medical University.

\section{In-house real-time reverse transcription-polymerase chain} reaction, tissue microarrays, and immunohistochemistry

Fresh HCC tissue specimens and adjacent normal hepatic tissue specimens were prepared to extract total RNA by employing the AxyPrep Multisource Total RNA Miniprep Kit (AP-MN-MS-RNA-250G, AXYGEN, China). RNA was reversely transcribed into cDNA using the 'Takara PrimeScript RT reagent' kit (Takara, Nanning, People's Republic of China, as we previously reported [26]. Betaactin $(A C T B)$ was used as the internal reference gene (forward sequence: " 5 '-CAGGCACCAGGGCGTGAT $-3^{\prime \prime}$; reverse sequence: " $5^{\prime}$-TAGCAACGTACATGGCTG GG-3'") [27]. The primer of the target gene was designed on the mRNA sequence between the two gRNA binding sites (forward sequence: $5^{\prime}$-GTGACCATGACGATGTCC TCC-3'; reverse sequence: $5^{\prime}$-GTTCCCCAGATCGGG ATGTC-3'). An algorithm of $2^{-\Delta c q}$ was used to determine the relative mRNA expression level of LPCAT1 [28-30].

The protein expression status of LPCAT1 in HCC was detected by conducting IHC, which was based on tissue microarrays. All the experimental operations concerning tissue microarrays and IHC staining follow the instructions of the manufacturer. The interpretation of staining intensity was performed by three expertized pathologists, according to the previously reported criteria [31].

\section{In-silico data mining of HCC RNA sequencing and gene chip datasets}

The mRNA expression profiles of HCC submitted before October 2020 were queried and filtered from public biomedical databases, including Gene Expression Omnibus, ArrayExpress, The Cancer Genome Atlas, Sequence Read Archive, The Genotype-Tissue Expression, and scientific 
literature. The inclusion criteria were as follows: (I) the subjects included in the studies should be Homo sapiens; (II) the patients should be diagnosed with HCC; (III) the platform information should be provided to annotate each probe. The exclusion criteria were as follows: (I) the subjects were animals; (II) valid data related to LPCAT1 expression was not presented; (III) duplicated data was reported; (IV) publications were in the form of case reports, reviews, and editorials. Three authors performed datasets screening independently, and an agreement was reached. $\mathrm{A} \log _{2}(\mathrm{x}+1)$ transformation was considered if the expression matrices had not been normalized. The included datasets were classified according to their affiliated sequencing agencies. Those datasets that belonged to the same sequencing agency were integrated into a larger expression matrix, and the inter-batch differences were removed by using Limma-Voom and sva packages in $\mathrm{R}$ v.3.6.1 software. The batch-removed expression matrices were prepared for gene analysis.

\section{In vitro cellular biological experiments}

Construction of recombinant plasmids with the clustered regularly interspaced short palindromic repeats (CRISPR)/ associated protein 9 (Cas9) system

We designed two gRNA sequences gRNA1: 5'-GGCGTC GAAGTAGGACGAGTG-3' and gRNA2: 5'-GTCTGA CCGGGACACGAACAC-3'. By synthesizing the oligoDNAs of these gRNAs and annealing them, then cloning them into backbone vectors 'pSpCas9(BB)-2A-Puro (PX459) V2.0' (Plasmid \#62988, addgene), two gRNAexpressing plasmids were formed [32].

\section{Transfection with plasmids}

HCC-derived cells (SMMC-7721 and Huh7) were stably transfected through lipo3000. After transfection for $24 \mathrm{~h}$, the complete medium containing $2 \mu \mathrm{g} / \mathrm{ml}$ puromycin was screened for $48 \mathrm{~h}$, and then the medium was transferred to the complete medium without puromycin to continue the culture. Transfected cells were used for further experiments.

\section{Knockout effect verification}

RT-qPCR was used to check the gene knockout efficiency. $A C T B$ was used in combination as the internal reference gene. The primers of $A C T B$ and the target gene were aforementioned in the "In-house real-time reverse transcription-polymerase chain reaction, tissue microarrays, and immunohistochemistry" section.

\section{Cell viability}

Proliferation assay was used to detect the effect of LPCAT1 knockout on cell viability. Total $2 \times 10^{3}$ transfected cells were seeded in each well of a 96-well plate.
The cells were counted every individual day and lasted for five days. The numbers of cells between the experimental group and the blank control group were compared, and the cell growth curve was drawn.

\section{Migration assay}

Cell migration ability was measured using a transwell cell in a 24-well plate (corning, Costar 3422). A total of $100 \mu \mathrm{l}$ medium $\left(0.5 \times 10^{5}\right.$ cells $)$ was added to the upper chamber, and $500 \mu \mathrm{l}$ of medium was added to the lower chamber. The mediums in the upper and lower chambers were DMEM containing 5\% FBS. After incubation at $37{ }^{\circ} \mathrm{C}$ for $24 \mathrm{~h}$, the cells on the upper surface of the chamber were gently wiped with cotton swabs [20], washed twice with phosphate buffered solution (PBS), fixed in methanol for $30 \mathrm{~min}$, dried and dyed in $0.1 \%$ crystal violet for $30 \mathrm{~min}$, and washed with PBS to remove the excess dye solution. The dyeing solution was observed under a microscope after drying. A total of five fields per chamber were subjected for the migrated cell counting.

\section{Invasiveness assay}

Cell invasiveness was measured using a transwell cell in a 24-well plate (corning, Costar 3422). Matrigel (BD, 356234) was diluted with serum-free DMEM. The ratio of Matrigel to DMEM was 1:8. $60 \mu \mathrm{l}$ of the diluted Matrigel was added to the chamber. After incubation at $37{ }^{\circ} \mathrm{C}$ for $1 \mathrm{~h}$, the chamber was taken out and the liquid was aspirated before DMEM was used to wash it twice. A total of $100 \mu \mathrm{l}$ of serum-free DMEM $\left(0.5 \times 10^{5}\right.$ cells $)$ was added into the chamber, and $500 \mu \mathrm{l}$ of DMEM containing $5 \%$ FBS was added into the lower chamber and cultured at $37^{\circ} \mathrm{C}$ for $24 \mathrm{~h}$. A total of five fields per chamber were subjected for the invaded cell counting.

\section{Cell cycle and apoptosis}

The cell cycle distribution and apoptotic rate of the LPCAT1-knockout group and control blank group were detected using a flow cytometer in SMMC-7721 and Huh7 cells.

\section{Prospective molecular mechanisms of LPCAT1 in HCC}

HCC differential expression genes (DEGs, $\mid \log _{2}$ FoldChange $\mid>1$ and $\mathrm{P}<0.05)$ were initially filtered from the batch-removed expression matrices by using the Limma package in $\mathrm{R}$ software v3.6.1. Standardized mean differences $(\mathrm{SMD},|\mathrm{SMD}|>0$ and $\mathrm{P}<0.05)$ were then quantitively calculated to identify DEGs, which were more reliable [33]. LPCAT1 co-expressed genes (CEGs, Pearson Correlation Coefficient, $|\mathrm{PCC}| \geq 0.3$ and $\mathrm{P}<0.05)$ were filtered from the batch-removed expression matrices. Specifically, one gene with a PCC value $\geq 0.3$ were defined as positive CEGs, while genes with a PCC 
value $\leq-0.3$ were defined as negative CEGs. DEGs and CEGs were intersected to analyse the clustered Gene Ontology (GO) terms, Disease Ontology (DO), reactome, and Kyoto Encyclopaedia of Genes and Genomes (KEGG) pathways by using the clusterprofiler package. The Search Tool for the Retrieval of Interacting Genes (STRING) database was used to analyse protein-protein interaction (PPI) [34]. Functional module analysis was conducted by using an algorithm of vertex-weighted MCODE in Cytoscape v3.6.1 software [35]. The hub gene in the PPI network was identified using Cytoscape. The genetic alterations and mutations of specific genes enriched in the first ranked KEGG pathways were analysed using the maftools package and cBioportal [36].

\section{Potential small molecule agents for HCC treatment}

Although increasing novel cancer targets are reported, their practical implications are abrogated by poor druggability of such targets [37]. However, a connectivity map (Cmap) provides an opportunity to repurpose Food and Drug Administration-approved drugs according to specific gene signatures, which is a potential strategy for precision treatment of cancer [38-40]. By targeting at the gene regulatory network of the DEGs that were coexpressed with LPCAT1 in HCC, several small molecule drugs for HCC treatment were forecasted.

\section{Data analysis}

All the data were analysed under IBM Statistical Product and Service Solutions Statistics v19.0, STATA v12.0, and $\mathrm{R}$ v3.6.1 software. An independent samples t-test was used to compare the LPCAT1 expression data in HCC tissues and non-tumour hepatic tissues. To combine SMD and hazard ratio (HR), a fixed-effect model was first considered when the $\mathrm{I}^{2}<50 \%$. A randomized-effect model should be used to handle the significant heterogeneity $\left(\mathrm{I}^{2} \geq 50 \%\right)$. Sensitive analysis was adopted to detect the source of heterogeneity. Begg's funnel plot was used to explore publication bias. The receiver operating characteristic curve (ROC) was plotted to determine the sensitivity and specificity. Summary receiver operating characteristic (SROC) curves were utilized to describe the area under the curve (AUC), pooled sensitivity, and pooled specificity. The likelihood ratio was used to determine the discriminatory ability of LPCAT1 between $\mathrm{HCC}$ and non-HCC tissues. Kaplan-Meier analysis was performed to assess the prognostic value of LPCAT1. $\mathrm{P}<0.05$ signified significance.

\section{Results}

Increased LPCAT1 mRNA expression in HCC tissues

The result of in-house RT-qPCR indicated that LPCTA1 mRNA was dominantly increased in 204 HCC tissue specimens as opposed to 204 non-tumour liver tissue specimens (Fig. 1a; Additional file 1: Table S1). Increased LPCAT1, however, possessed a weak ability to discriminate between HCC and normal liver tissues (Fig. 1b), which was due to a relatively small sample size. Eight out of ten external datasets confirmed the upregulated LPCAT1 expression level (Additional file 2: Figure S1), where $L P C A T 1$ showed a moderate or strong ability to discriminate between HCC and normal liver tissues in four subsets. The basic information of such datasets was

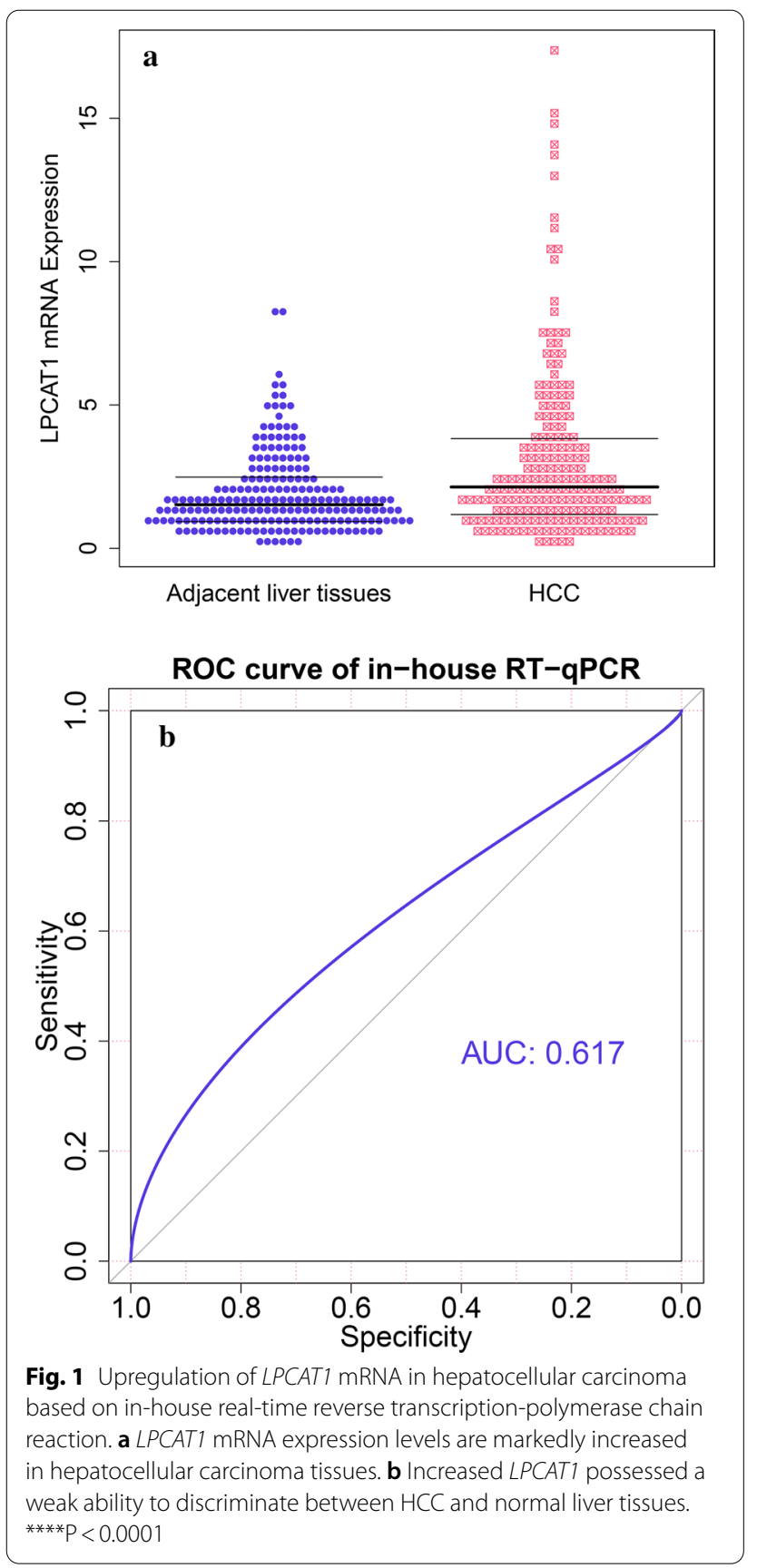


presented in Additional file 1: Table S1 and Additional file 3: Table S2. Collectively, a combined SMD value of $0.63[0.35,0.90]$ integrated in-house RT-qPCR results and external datasets and verified the increased mRNA expression levels of LPCAT1 in 3715 HCC tissue samples in comparison with 3105 non-HCC tissue samples (Additional file 4: Figure S2a). A randomized-effects model was utilized to handle the significant heterogeneity $(\mathrm{P}<0.01)$. The result of the sensitive analysis failed to identify a heterogeneity source (Additional file 4: Figure S2b). No publication bias was detected (Additional file 4: Figure
S2c). The result of SROC indicated a moderate ability of LPCAT1 in differentiating between HCC and non-HCC $(\mathrm{AUC}=0.80[0.77,0.84]$; sensitivity $=0.59[0.42,0.74]$; specificity $=0.85[0.74,0.92]$; combined diagnostic likelihood ratio positive $=3.98$ [2.32, 6.82]; and combined diagnostic likelihood ratio negative $=0.48[0.34,0.69]$ ) (Additional file 5: Figure S3a-c).

\section{Increased LPCAT1 protein expression level in HCC tissues}

As opposed to normal liver tissue specimens (Fig. 2ab), LPCAT1 protein expression levels were markedly

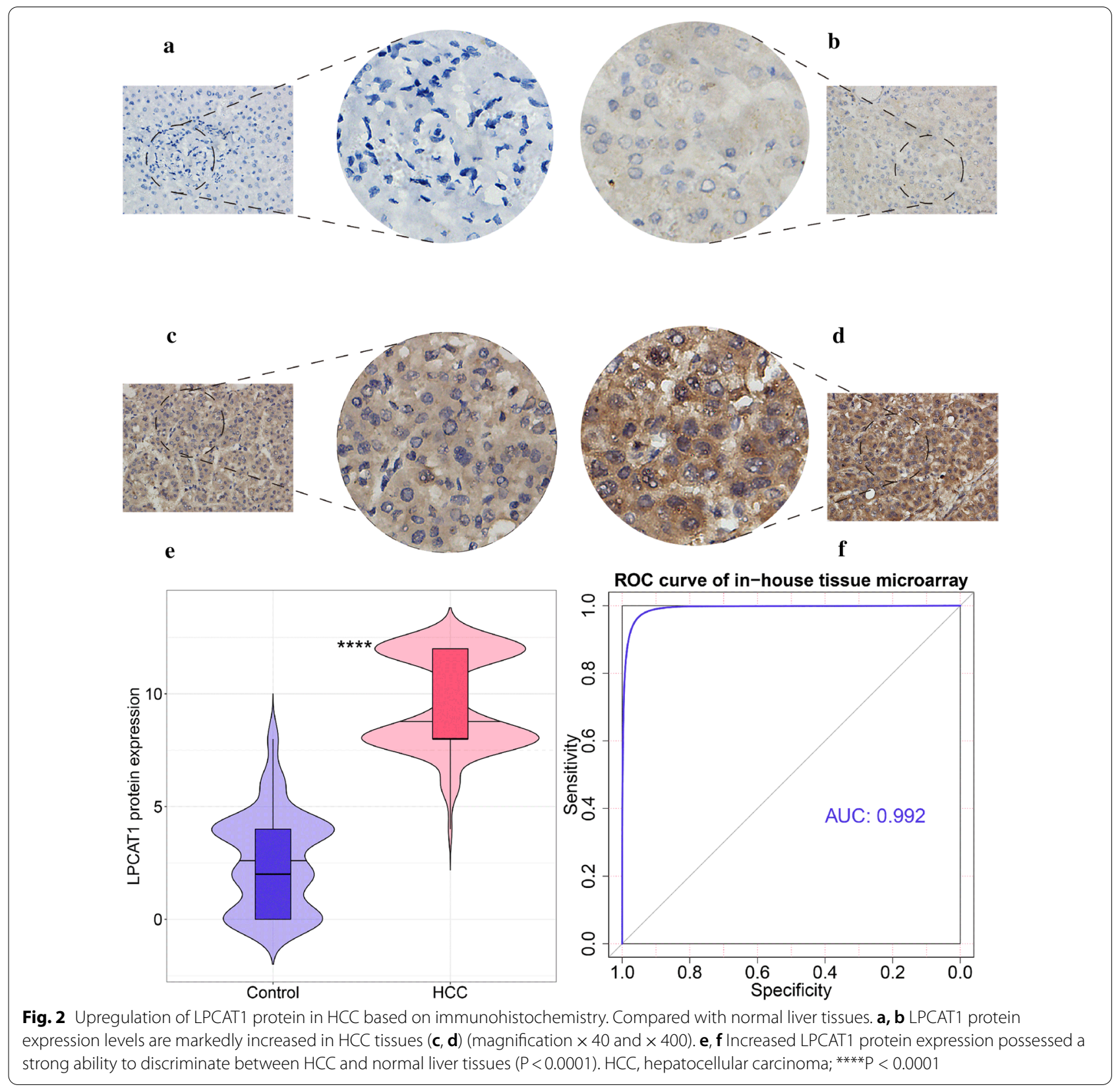


increased in the HCC tissue specimens (Fig. 2c-d), which was consistent with LPCAT1 mRNA expression. Increased LPCAT1 protein expression possessed a strong ability to discriminate between HCC and normal liver tissues (Fig. 2e-f).

Poor prognosis of HCC patients with LPCAT1 upregulation The result of Kaplan-Meier survival analysis indicated that higher LPCAT1 expression forecasted worse overall survival outcomes in HCC patients (Fig. 3a). Increased LPCAT1 expression patterns varied from gender (male: $3.28 \pm 3.10$ vs. female: $1.73 \pm 1.66 ; \mathrm{P}=0.001)$, age $(<60$ : $3.33 \pm 3.22$ vs. $\geq 60: 2.17 \pm 1.65 ; \mathrm{P}=0.002)$ and microvascular tumour thrombus (with thrombus: $3.50 \pm 3.20$ vs. without thrombus: $2.64 \pm 2.71 ; \mathrm{P}=0.040$ ) (Fig. 3b-d). External $\mathrm{HCC}$ cohorts were used to confirm the prognostic value of LPCAT1 in HCC (Additional file 6: Figure S4a). The GSE10143 cohort was probed to be the primary
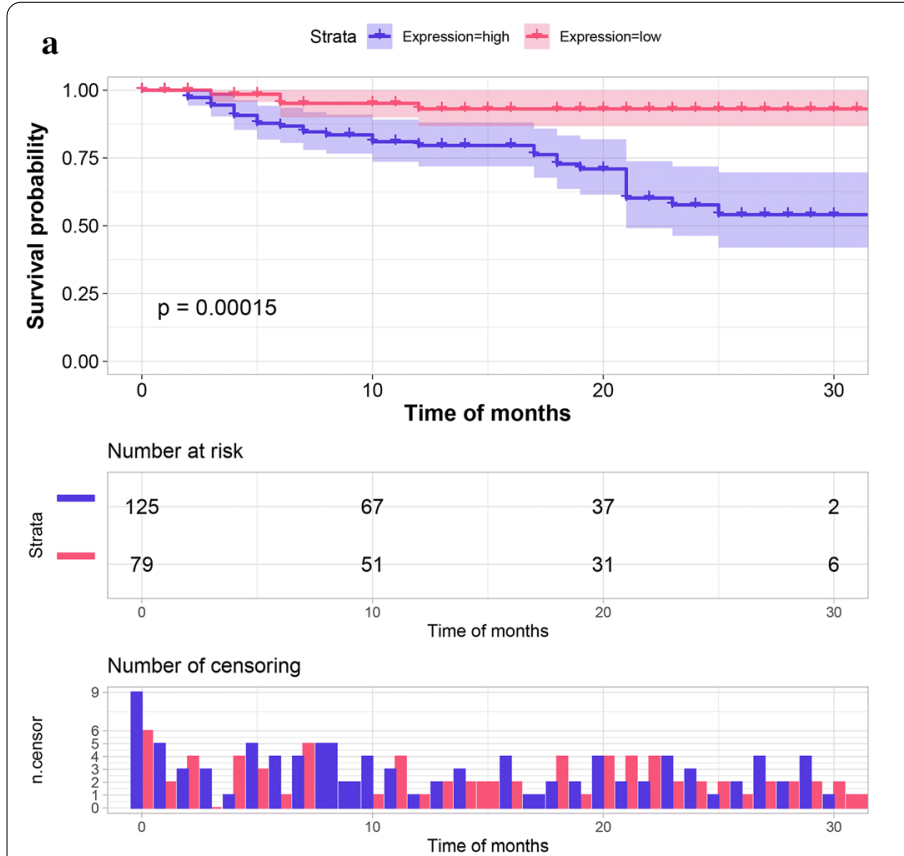

b

c
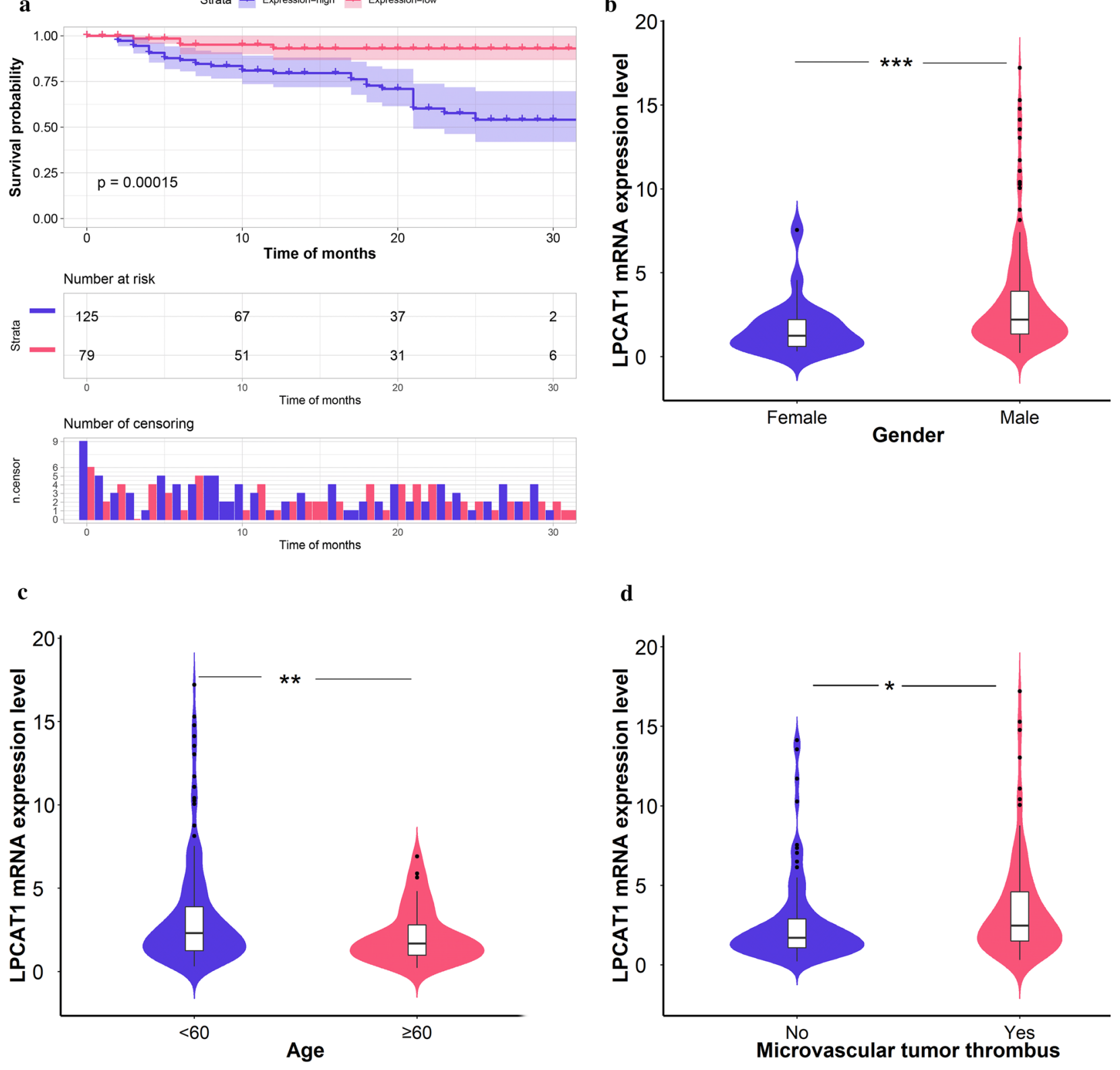

d

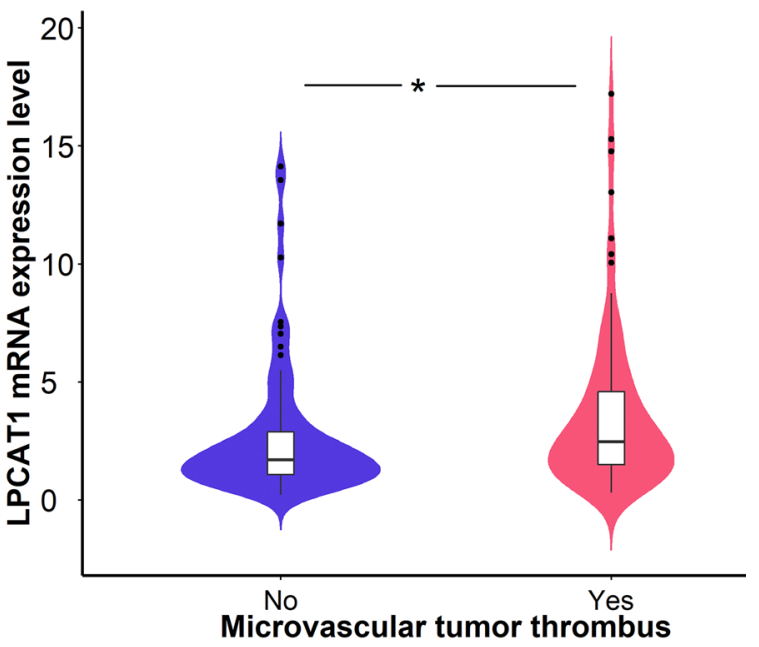

Fig. 3 Increased expression of LPCAT1 is correlated with poor prognosis of HCC patients. a Higher LPCAT1 expression predicts worse overall survival outcomes. b-d Among HCC patients, elevated expression levels of LPCAT1 were significantly different in terms of gender, age, and microvascular tumor thrombus. ${ }^{* *} \mathrm{P}<0.001 ;{ }^{* *} \mathrm{P}<0.01 ;{ }^{*} \mathrm{P}<0.05$. HCC, hepatocellular carcinoma 
source of heterogeneity and had been removed (Additional file 6: Figure S4b). Collectively, a combined HR value of 2.21 [1.64, 2.99] indicated that LPCAT1 might serve as an independent risk factor for HCC (Additional file 6: Figure S4c).

\section{Establishment of LPCAT1 knockout HCC cells}

To reveal the cellular biological functions of LPCAT1, LPCAT1 knockout cells were successfully established and were transfected into two HCC-derived cell lines (i.e., SMMC-7721 and Huh7) [20]. The result of the RT-qPCR verification experiment indicated lower LPCAT1 mRNA expression levels in LPCAT1 knockout SMMC-7721 cells $(\mathrm{P}=4.0 \mathrm{E}-05)$ and LPCAT1 knockout Huh7 cells $(\mathrm{P}=3.3 \mathrm{E}-06)$ than those in the control group (Fig. 4a-b).

\section{Inhibited proliferation, migration, and invasion abilities in LPCAT1 knockout HCC cells}

It was found that compared with the blank control group, LPCAT1 knockout significantly hampered cellular growth in SMMC-7721 and Huh7 cells (Fig. 4c-d). Therefore, LPCAT1 might promote the proliferation of HCC cells. Compared with the LPCAT1 non-knockout transfected cells in the two cell lines, there was a significant decrease in the number of penetrating LPCAT1 knockout SMMC-7721 and Huh7 cells (Figs. 5, 6). Therefore, LPCAT1 might enhance the migration and invasion abilities of SMMC-7721 and Huh7 cells.

\section{Cell cycle and apoptosis of LPCAT1 knockout cells}

It was observed that compared with the distribution of $56.45 \%$ cells in the control group, about $53.32 \%$ of LPCAT1 knockout cells were distributed in the G0/ G1 period. Additionally, about $31.16 \%$ of LPCAT1 knockout cells were distributed in the $\mathrm{S}$ period, higher than that of $28.45 \%$ cells in the control group (Fig. $4 \mathrm{e}-\mathrm{g}$ ). Therefore, LPCAT1 knockout might arrest the cell cycle at the $\mathrm{S}$ phase. Additionally, the apoptotic rates of LPCAT1 knockout SMMC-7721 cells slightly increased when compared with the blank control group (Fig. $4 \mathrm{~h}-\mathrm{j}$ ). However, the statistical significance cannot be determined because of a lack of duplicate samples.
Prospective signal transduction pathways of LPCAT1 in HCC A total of 5399 HCC DEGs were identified, containing 3736 overexpressed DEGs and 1663 under expressed DEGs, all with significant SMD. Additionally, a total of 1223 LPCAT1 CEGs were determined, including 564 positive CEGs and 659 negative CEGs. Upregulated DEGs were intersected by positive CEGs, and 473 genes were obtained whose expression level was increased in HCC and were positively correlated with LPCAT1. Downregulated DEGs were intersected by negative CEGs, and 367 genes were obtained. Enrichment analysis indicated that the upregulated LPCAT1 CEGs were dominantly clustered in the cell cycle pathway according to the KEGG (Additional file 7: Figure S5a) and reactome (Additional file 7: Figure S5b). Additionally, embryonal cancer was determined to be the most enriched disease (Additional file 7: Figure S5c). Moreover, mitotic nuclear division, cell-substrate adherens junction, and cadherin binding were found to be the most prominent "Biological Process," "Cellular Component," and "Molecular Function," respectively (Additional file 8: Figure S6). On the other hand, the downregulated LPCAT1 CEGs were primarily clustered in chemical carcinogenesis (Additional file 9: Figure S7a) and biological oxidations (Additional file 9: Figure S7b). Accordingly, inherited metabolic disorder was found to be the most associated disease (Additional file 9: Figure S7c). Furthermore, small molecule catabolic process, plasma lipoprotein particle, and monooxygenase activity were identified as the most aggregated GO terms according to the downregulated genes negatively related to LPCAT1 in HCC (Additional file 10: Figure S8). The mutation oncoplot of the cell cycle and chemical carcinogenesis is presented in Additional file 11: Figure S9a-b.

\section{Construction of the genetic regulatory network of LPCAT1 in $\mathrm{HCC}$}

LPCAT1's genetic regulatory network in HCC was constructed based on the intersected genes that had been described in the "Prospective signal transduction pathways of LPCAT1 in HCC" section (Fig. 7). Two functional modules (i.e., Cluster 1 and Cluster 2) were identified for the upregulated CEGs and downregulated CEGs, respectively. PPI of the cell cycle and chemical carcinogenesis pathways were explored further. $C C N B 1$, which was significantly correlated with LPCAT1 (Spearman $\mathrm{R}=0.58, \mathrm{P}=2 \mathrm{E}-48$ ), was established as the hub gene in the cell cycle pathway. Moreover, a total of three core genes were established

(See figure on next page.)

Fig. 4 LPCAT1 induced the proliferation of Huh7 and SMMC-7721 cells. The LPCAT1-knockout Huh7 and SMMC-7721 cells were constructed by using the clustered regularly interspaced short palindromic repeats (CRISPR)/associated protein 9 (Cas9) system. $\mathbf{a}$, $\mathbf{b}$ Validated the transfection efficiency of LPCAT1-knockout group and control group in Huh7 and SMMC-7721 cell lines, respectively. $\mathbf{c}$, $\mathbf{d}$ Proliferation assay was conducted to evaluate the effect of LPCAT1-knockout on cellular proliferation. $\mathbf{e}-\mathbf{j}$ Flow cytometry was used to detect the cycle distribution and apoptosis of LPCAT1-knockout SMMC-7721 cells. ${ }^{* * * P}<0.0001 ;{ }^{* * * P}<0.001 ;{ }^{* *} P<0.01$ 

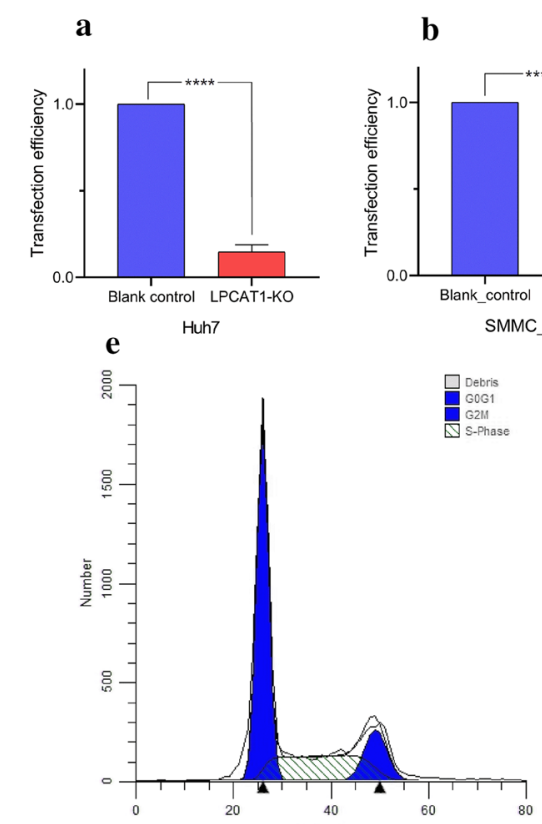

h

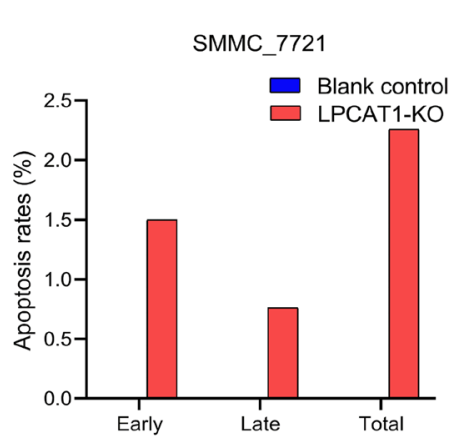

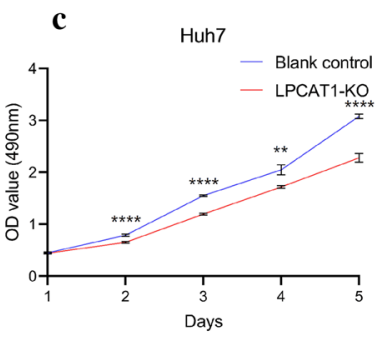

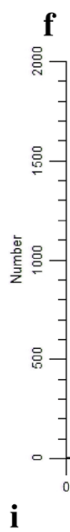

I첪
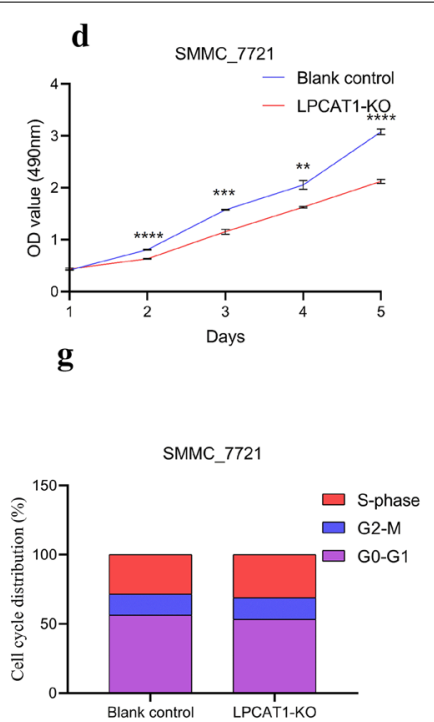
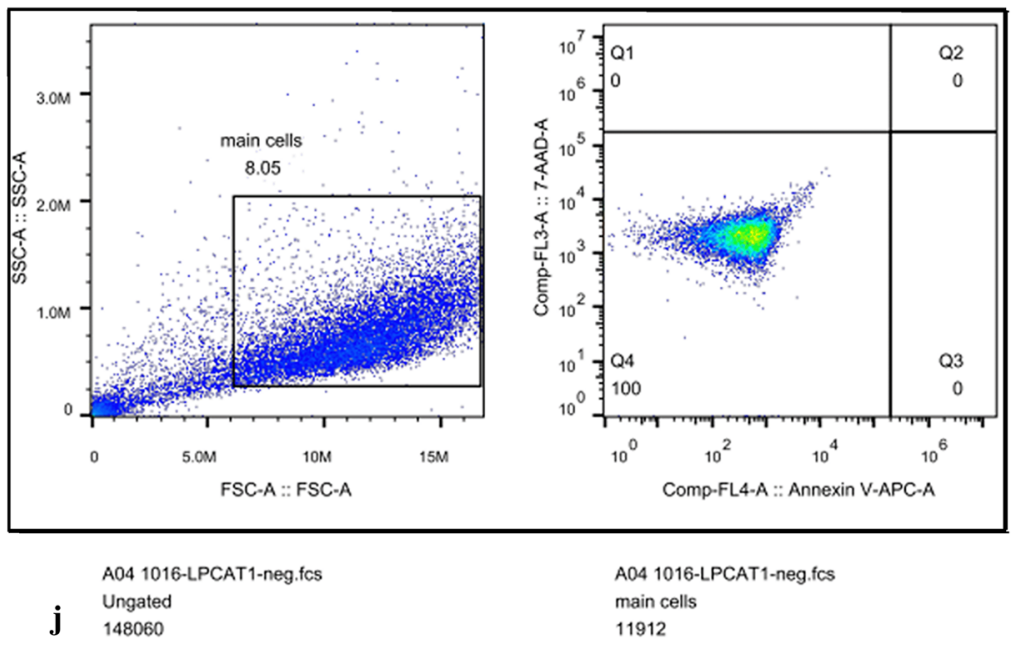

A04 1016-LPCAT1-neg.fCS main cells 11912
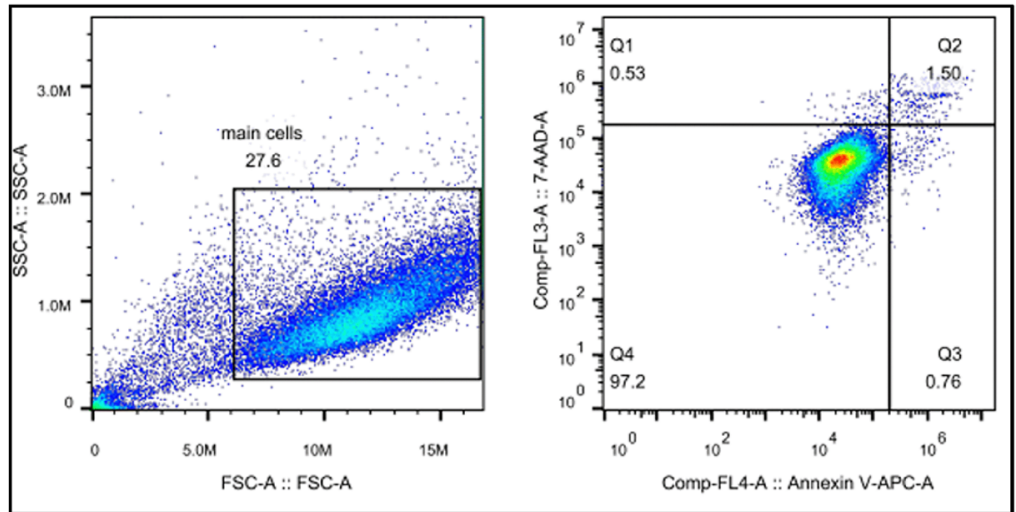

B04 1016-LPCAT1-sam.fCS

Ungated

B04 1016-LPCAT1-sam.fCS main cells 22979

Fig. 4 (See legend on previous page.) 

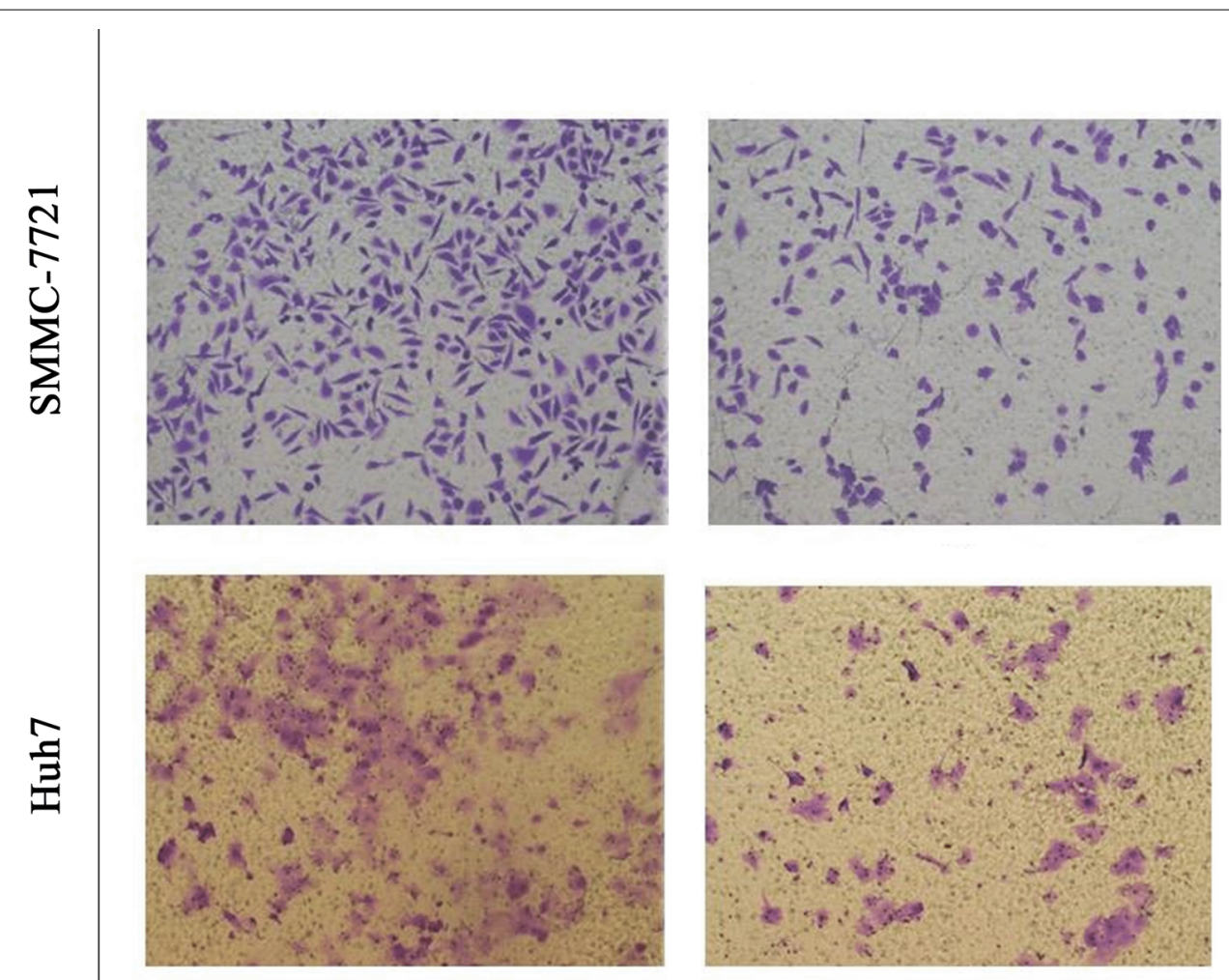

LPCAT1-NC

LPCAT1-KO

$\square$ LPCAT1-NC

$\square$ LPCAT1-KO

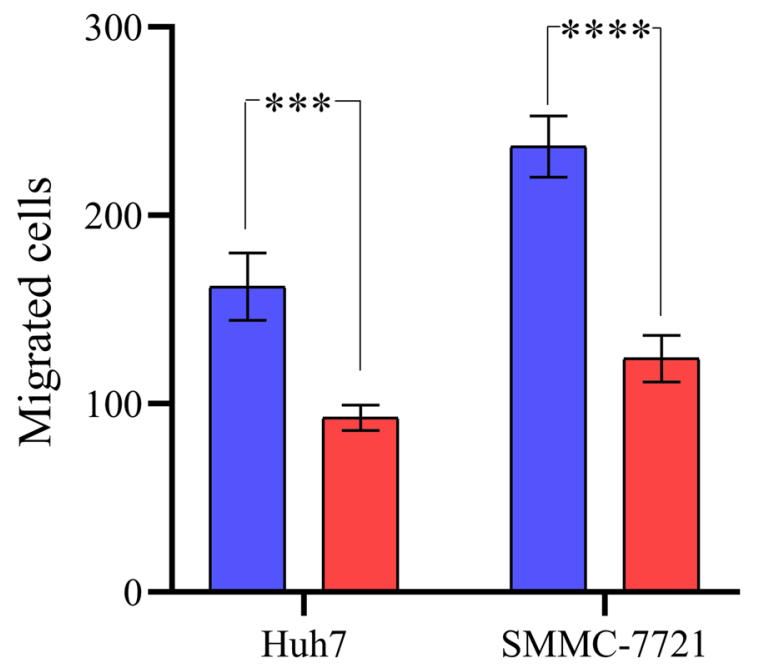

Fig. 5 The ability of cell migration to LPCAT1-knockout group and control group in SMCC-7721 and Huh7 cell lines. LPCAT1 might promote the migration of SMCC-7721 and Huh7 cells. ${ }^{* * *} \mathrm{P}<0.0001$; ${ }^{* * *} \mathrm{P}<0.001$ 

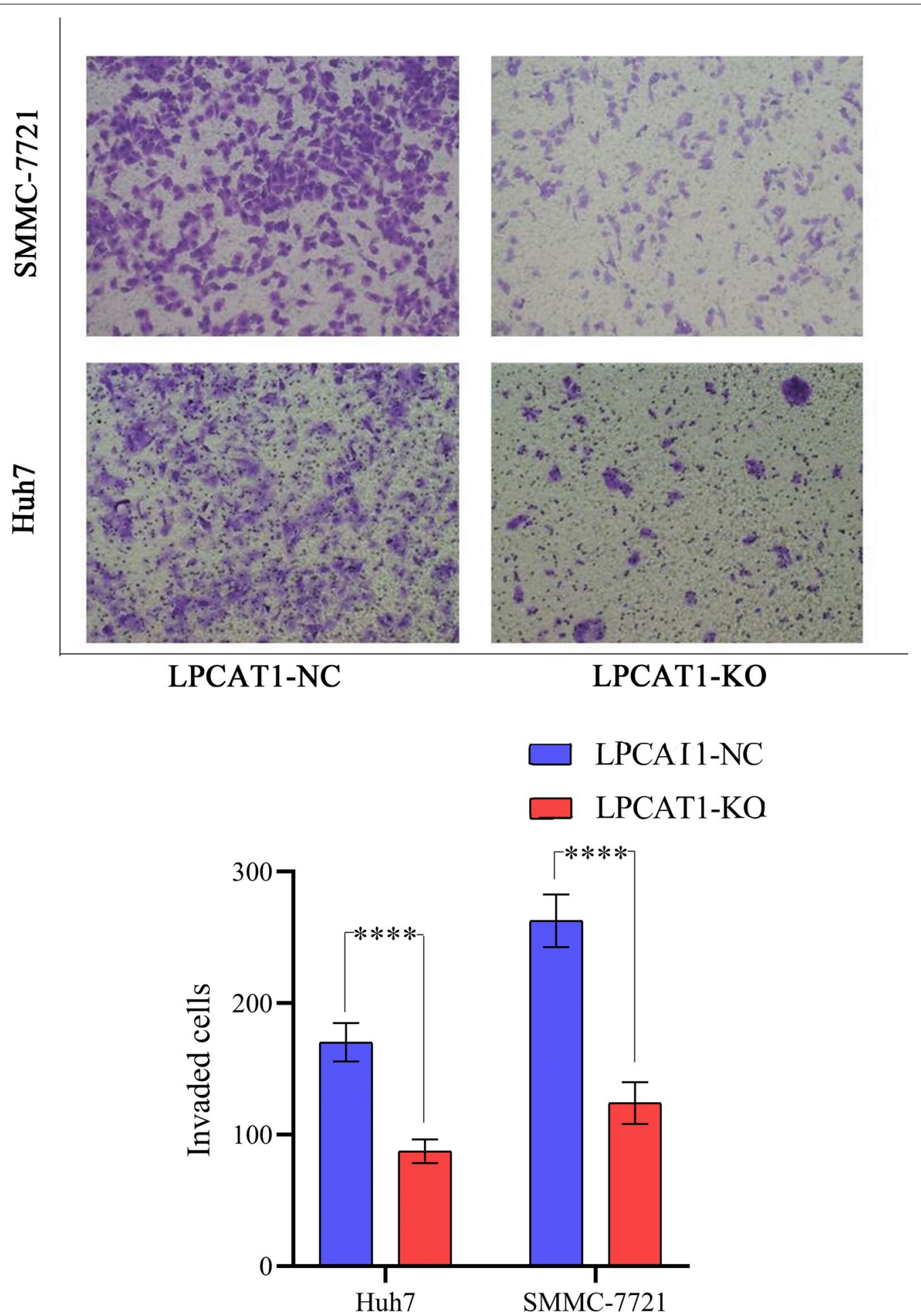

Fig. 6 The ability of cell invasion to LPCAT1-knockout group and control group in SMCC-7721 and Huh7 cell lines. LPCAT1 might promote the invasion of SMCC-7721 and Huh7 cells. ${ }^{* * *} \mathrm{P}<0.0001$ 


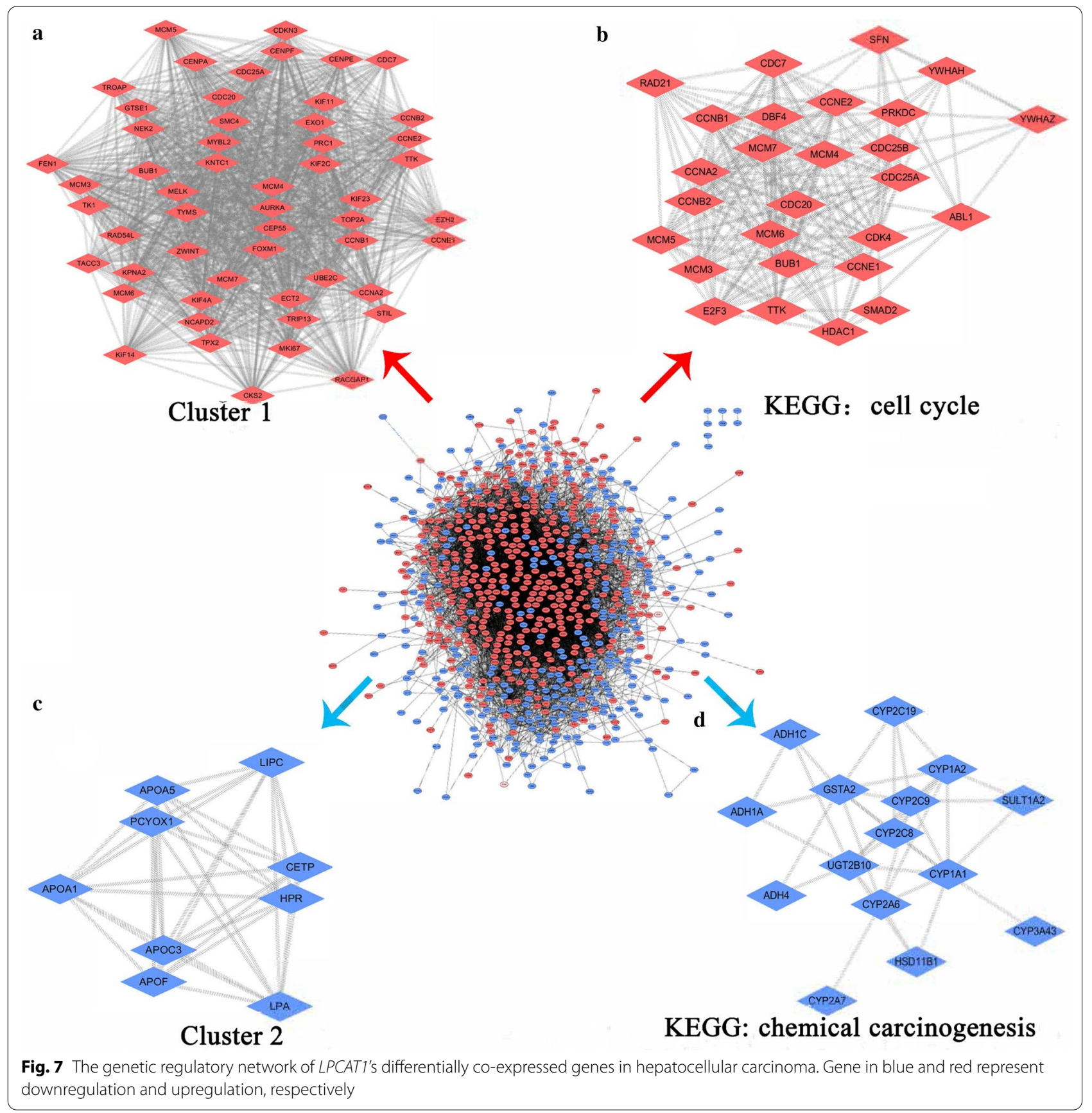

in the chemical carcinogenesis pathway, containing UGT2B10 (Spearman $\mathrm{R}=-0.50, \mathrm{P}=3.2 \mathrm{E}-34$ ), GSTA2 (Spearman $\mathrm{R}=-0.30, \mathrm{P}=2.4 \mathrm{E}-12$ ), and $C Y P 1 A 1$ (Spearman $\mathrm{R}=-0.37, \mathrm{P}=3.1 \mathrm{E}-18$ ).

\section{Potential therapeutic agents for $\mathrm{HCC}$ by targeting at LPCAT1's regulatory network}

By constructing the gene regulatory network based on the DEGs related to LPCAT1 in HCC, several potential therapeutic agents for $\mathrm{HCC}$ were identified by targeting at LPCAT1's regulatory network (Table 1). The molecular formulas of such drugs are presented in Additional file 12: Figure S10. Surprisingly, the anti-HCC effects of LY-294002 [41], trichostatin A [42], sirolimus [43], 


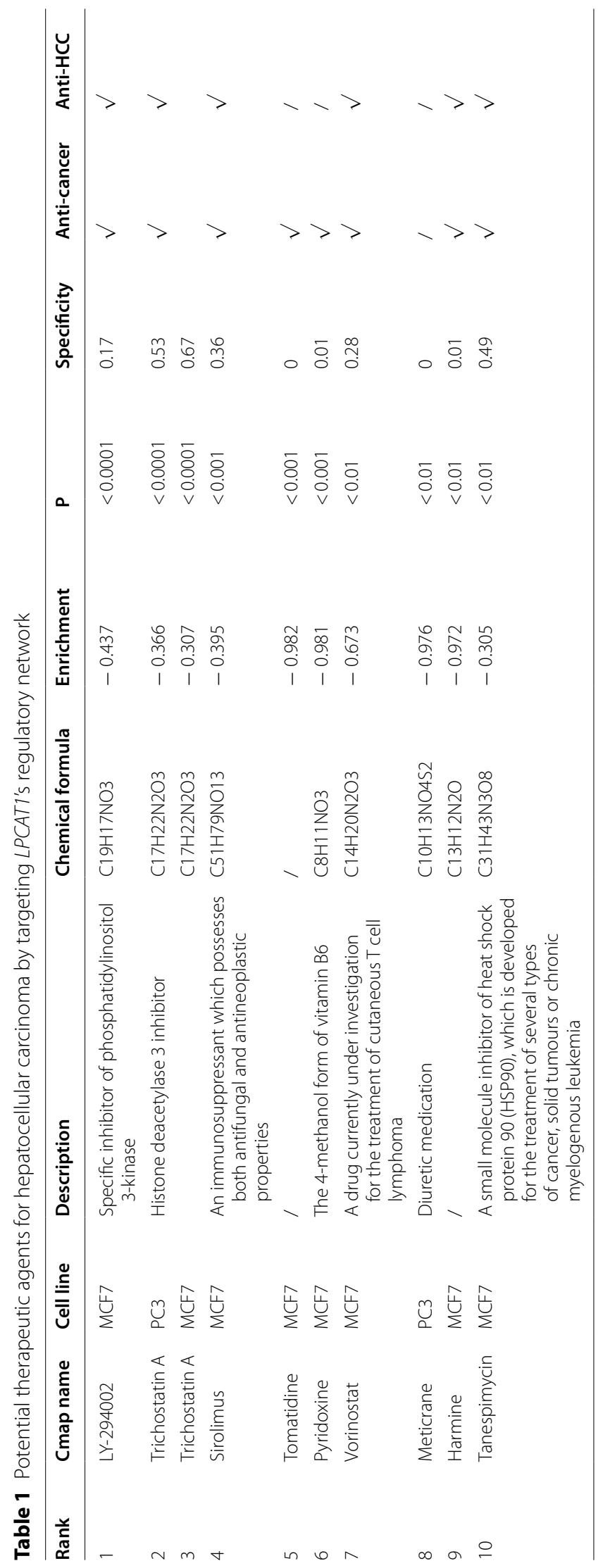


vorinostat [44], and harmine [45] have been demonstrated by in vitro or in vivo experiments.

\section{Discussion}

Lipid metabolisms have been verified to play profound roles in the onset and deterioration of HCC through lipid metabolic reprogramming and metabolism rearrangements [46-48]. Mounting evidence demonstrated that LPCAT1 participates in the pathophysiological process of cancer by dysregulating lipid metabolisms $[16,48]$. In this study, the authors found that increased LPCAT1 induced the proliferation, migration, and metastasis of SMMC7721 and Huh7 cells. A regulatory network of LPCAT1 was constructed to analyse the molecular mechanisms behind the initiation and progression of HCC. Several small molecule drugs were forecasted by targeting at the network.

There was only one small study that elucidated the upregulation of LPCAT1 in HCC [22]. By contrast, the present study highlights the biological functions of LPCAT1 and verifies its upregulated expression patterns in 3715 HCC specimens as opposed to 3105 nonHCC specimens. Furthermore, the prognostic value of LPCAT1 was comprehensively assessed by in-house and external HCC cohorts. More importantly, the cell cycle and chemical carcinogenesis were identified as the two most enriched signalling pathways. Potential drugs targeting at LPCAT1 in HCC were predicted.

LPCAT1 mRNA and protein were abundant in HCC tissues. In-house RT-qPCR, immunohistochemistry, external RNA sequencing, and gene chip were used to confirm the increased expression levels of LPCAT1 in HCC tissues. A SMD index was used to combine the effect size, which further supported the upregulation of LPCAT1 $(\mathrm{SMD}=0.63[0.35,0.90])$. Therefore, the results of the present study are more convincing. Additionally, increased LPCAT1 presages poor prognosis of HCC patients. Higher LPCAT1 was correlated with worse overall survival conditions in $125 \mathrm{HCC}$ patients, suggesting LPCAT1 might be a potent risk factor in HCC (combined $\mathrm{HR}=2.21$ [1.64, 2.99]). Also, LPCAT1 expression shows an ascending pattern in male, age $<60$, and microvascular tumour thrombus HCC patients. Collectively, it is conceivable that upregulated LPCAT1 might play crucial roles in the unfavourable prognosis of HCC.

LPCAT1 might promote HCC progression by accelerating cell growth, migration, and metastasis. LPCAT1 knockout SMMC-7721 and Huh7 cells were established using the CRISPR/Cas9 system [49]. The proliferation, migration, and metastasis abilities of LPCAT1 knockout transfected SMMC-7721 and Huh7 cells were significantly hampered, thus indicating that LPCAT1 might participate in the deterioration of HCC cells by promoting the mechanisms of cell growth, migration, and metastasis. This hypothesis can be supported by a previous study, where only one HCC-derived cell line was used [22]. Interestingly, another study reported that LPCAT1 is required for the miRNA-205-induced proliferation of SMMC-7721 cells [50]. Furthermore, LPCAT1 knockout might arrest the cell cycle at the S phase and increase the cell apoptotic rates in SMMC-7721 cells, which was reported for the first time. Therefore, the authors speculate that LPCAT1 might induce the DNA synthesis process and abrogate cell apoptosis to survive HCC cells. However, the influence LPCAT1 has on the cell cycle distribution and the apoptotic rate of HCC cells has not been verified by duplicate samples. More experimental evidence must be supplemented in the future to support the findings in the present study.

Since LPCAT1 was important in HCC initiation and progression, the authors sought to shed light on its potential molecular mechanisms underlying HCC. A genetic regulatory network of LPCAT1 was constructed, where upregulated LPCAT1 CEGs were prominently clustered in the cell cycle pathway. As is known, aberrant regulation of the cell cycle induces uncontrollable cell proliferation and handles various cancers, including HCC [51-53]. The result of flow cytometry unveils an intimate association between LPCAT1 and cell cycle regulation; however, the precise regulatory mechanisms had never been studied before. In this study, upregulated LPCAT1 CEGs were found to participate in mitotic nuclear division, regulation of mitotic cell cycle phase transition, cell cycle checkpoints, and mitotic G1-G1/S phases in terms of GO and reactome. During mitotic nuclear division, the primary event of the $M$ phase, mitotic genes are prone to be activated when the chromatin structures change, which creates favourable conditions for the proliferation of HCC cells [54]. Interestingly, CCNB1, the hub gene in the cell cycle pathway, encodes an important regulatory protein in mitosis (cyclin B1). Increased cyclin B1, prominently working as a "switch" in the process of cell cycle, results in malignant proliferation of HCC cells by binding to cyclin-dependent kinases [55]. Furthermore, there was a positive association between the mRNA expression levels of LPCAT1 and CCNB1 (Spearman $\mathrm{R}=0.58$, $\mathrm{P}=2 \mathrm{E}-48$ ), indicating that there might be a synergetic effect between LPCAT1 and CCNB1 on the pathogenesis of HCC. Additionally, the cell cycle checkpoint is verified to be one of the most important cell cycle regulatory mechanisms [56]. Nonetheless, dysregulation of the cell cycle checkpoint might lead to radio resistance of HCC cells, thus causing unfavourable prognosis in HCC patients [57]. Therefore, the upregulated LPCAT1 CEGs might induce abnormal regulation of the cell cycle and promote the development and deterioration of HCC. 
On the other hand, downregulated LPCAT1 CEGs provide important clues on metabolism mechanisms, which might propel chemical carcinogenesis. Downregulated LPCAT1 CEGs are involved in small molecule catabolic processes, plasma lipoprotein particles, and biological oxidations. Surprisingly, increased LPCAT1 is likely to induce metabolic disturbance and fuel the process of liver damage and carcinogenesis. In liver injury mouse models, LPCAT1 was upregulated in the carbon tetrachloride-treated mouse, along with increased saturated fatty acylphospholipid in liver tissues and peripheral plasma [58]. Furthermore, another study verified the lipid composition in Huh7 cells by using mass spectrometry imaging [22]. Collectively, it was conceivable that upregulated LPCAT1 might disturb the metabolism of lipids and promote liver carcinogenesis by co-expressing with downregulated CEGs. More studies are required to further confirm our hypothesis.

Moreover, several therapeutic drugs were successfully predicted by targeting at the genetic regulatory network of LPCAT1. As is known, the clinical efficiency of HCC patients was largely limited by chemotherapy resistance and liver dysfunction. It is urgently needed to develop more potent agents with hepatoprotective and antiHCC properties [59]. In the present study, LY-294002, trichostatin A, sirolimus, vorinostat, and harmine were forecasted to be potential small molecule drugs for treating HCC. LY-294002, an inhibitor of phosphatidylinositol 3-kinase, has proved useful in hampering HCC cell growth, migration, and invasion [60]. Additionally, histone deacetylase inhibitor trichostatin A has seen anti-HCC efficacy by activating cell death signalling cascades [61]. More importantly, a single-centre study demonstrated the favourable effect sirolimus has on HCC patients, whose survival time were longer than in the control group [62]. Therefore, such evidence indicates that repurposing FDA-approved small molecule drugs is a promising direction for $\mathrm{HCC}$ treatment. In vivo experiments and clinical trials will promote the applications of these small molecule drugs.

There are several limitations. First, a high level of heterogeneity was detected in the present study. The author used a randomized-effect model to minimize the influence of heterogeneity. In the future, more single cell RNA sequencing experiments will help to reduce intercellular heterogeneity. Second, the influence $L P C A T 1$ has on the cell cycle distribution and the apoptotic rate of HCC cells has not been verified by different cell lines. Third, the complicated molecular mechanisms and practical effects of the predicted small molecule drugs are unclear. However, the present study sheds light on several novel signalling pathways and reveals potential therapeutic directions for HCC. Further studies are needed to validate the conclusions.

\section{Conclusions}

LPCAT1 was upregulated and correlated with poor prognosis in HCC patients. Increased LPCAT1 fuelled HCC progression by promoting cell proliferation, migration, and metastasis. LPCAT1 co-expressed genes and HCC differentially expressed genes prominently participated in the cell cycle and chemical carcinogenesis pathways.

\section{Abbreviations}

HCC: Hepatocellular carcinoma; LPCAT1: Lysophosphatidylcholine acyltransferase 1; DMEM: Dulbecco's modified Eagle's medium; FBS: Fetal bovine serum; IHC: Immunohistochemistry; ACTB: Beta-actin; RT-qPCR: Real-time reverse transcription-polymerase chain reaction; CRISPR/Cas9: The clustered regularly interspaced short palindromic repeats/associated protein 9; PBS: Phosphate buffered solution; DEGs: Differential expression genes; CEGs: Co-expressed genes; SMD: Standardized mean differences; PCC: Pearson correlation coefficient; GO: Gene ontology; DO: Disease ontology; KEGG: Kyoto Encyclopaedia of Genes and Genomes; STRING: Search tool for the retrieval of interacting genes; PPI: Protein-protein interaction; Cmap: Connectivity map; HR: Hazard ratio; ROC: Receiver operating characteristic curve; SROC: Summary receiver operating characteristic; AUC: Area under the curve.

\section{Supplementary Information}

The online version contains supplementary material available at https://doi. org/10.1186/s12935-021-02130-4.

Additional file 1: Table S1: Fundamental information of the included datasets.

Additional file 2: Figure S1: Upregulation of LPCAT1 in hepatocellular carcinoma based on public datasets.

Additional file 3: Table S2: Basic statistics of the included datasets.

Additional file 4: Figure S2: Upregulation of LPCAT1 in 3715 hepatocellular carcinoma tissues compared with 3105 normal liver tissues. a Forest plot of standardized mean differences. b Sensitive analysis. c Begg's funnel plot.

Additional file 5: Figure S3: Potential clinical applications of LPCAT1 in HCC. LPCAT1 showed a moderate ability in differentiating HCC tissues from normal liver tissues according to a summary receiver operating characteristic curve, $\mathbf{b}$ forest plot of positive likelihood $\mathbf{c}$ forest plot of negative likelihood. HCC, hepatocellular carcinoma.

Additional file 6: Figure S4: Potential prognostic value of LPCAT1 in HCC. a Hazard ratios from in-house and external cohorts of HCC patients were combined. b GSE10143 was responsible for a high level of heterogeneity. c LPCAT1 may serve as an independent risk factor for HCC after the removal of GSE10143. HCC, hepatocellular carcinoma.

Additional file 7: Figure S5: Prospective molecular signaling transduction pathways based on upregulated genes positively related to LPCAT1 in hepatocellular carcinoma. a The Kyoto Encyclopedia of Genes and Genomes pathway. b The reactome pathway. c The disease ontology.

Additional file 8: Figure S6: Gene ontology enrichment catalogues based on upregulated genes positively related to LPCAT1 in hepatocellular carcinoma. Based on the upregulated genes positively related to LPCAT1 in HCC, mitotic nuclear division, cell-substrate adherens junction, and cadherin binding were found to be the most aggregated gene ontology terms. 
Additional file 9: Figure S7: Prospective molecular signaling transduction pathways based on downregulated genes negatively related to LPCAT1 in hepatocellular carcinoma. a The Kyoto Encyclopedia of Genes and Genomes pathway. b The reactome pathway. c The disease ontology.

Additional file 10: Figure S8: Gene ontology enrichment catalogues based on downregulated genes negatively related to LPCAT1 in hepatocellular carcinoma. Small molecule catabolic process, plasma lipoprotein particle, and monooxygenase activity were identified as the most aggregated gene ontology terms according to the downregulated genes negatively related to LPCAT1 in hepatocellular carcinoma.

Additional file 11: Figure S9: Genetic alterations to differentially expressed genes co-expressed with LPCAT1 in hepatocellular carcinoma. a Oncoplot of LPCAT1 alterations in hepatocellular carcinoma. $\mathbf{b}$ Oncoplot of differentially expressed genes co-expressed with LPCAT1 in cell cycle and chemical carcinogenesis pathway.

Additional file 12: Figure S10: Potential therapeutic agents for hepatocellular carcinoma by targeting LPCAT7's regulatory network. a LY-294002 (C19H17NO3). b Trichostatin A (C17H22N2O3). c Sirolimus (C51H79NO13). d Pyridoxine (C8H11NO3). e Vorinostat (C14H2ON2O3). f Harmine (C13H12N2O). g Tanespimycin (C31H43N3O8).

\section{Acknowledgements}

The authors appreciate people who are devoted to the availability of public biomedical databases.

\section{Authors' contributions}

Conceptualization, GC, R-QH, and Y-WD; methodology, GC, R-QH, Y-WD, L-JY, Z-GH, L-ML, and L-FL; validation, GC, R-QH; formal analysis, J-DL, X-FD; investigation, L-JY, Z-GH; resources, GC, R-QH, and Y-WD; data curation, GC; writingoriginal draft preparation, J-DL, X-FD; writing - review and editing, GC, R-QH, Y-WD, L-JY, Z-GH, L-ML, L-FL, and HY; visualization, J-DL, X-FD; supervision, GC, $\mathrm{R}-\mathrm{QH}$, and $\mathrm{Y}-\mathrm{WD}$; project administration, $\mathrm{GC}, \mathrm{R}-\mathrm{QH}$, and $\mathrm{Y}-\mathrm{WD}$; funding acquisition, GC, R-QH, and J-DL. All authors read and approved the final manuscript.

\section{Funding}

The present study was funded by Fund of National Natural Science Foundation of China (NSFC82160762); Natural Science Foundation of Guangxi, China (2018GXNSFAA294025); Guangxi Medical High-level Key Talents Training "139" Program (2020); Guangxi Medical University Training Program for Distinguished Young Scholars (2017); Medical Excellence Award Funded by the Creative Research Development Grant from the First Affiliated Hospital of Guangxi Medical University (2016); Guangxi Educational Science Planning Key Project (2021B167); Guangxi Higher Education Undergraduate Teaching Reform Project (2020JGA146); Guangxi Medical University Education and Teaching Reform Project (2019XJGZ04); China Postdoctoral Science Foundation (No. 2021M693804), Guangxi Zhuang Autonomous Region Health Committee Self-financed Scientific Research Project (Z20201147), and Guangxi Medical University Student Innovation and Entrepreneurship Training Program Project (202010598002)

\section{Declarations}

Ethics approval and consent to participate

The current research was ratified by the Ethics Committee of the First Affiliated Hospital of Guangxi Medical University.

\section{Consent for publication}

Not applicable.

\section{Availability of data and materials}

The datasets analysed during the current study are available in the Gene Expression Omnibus (https://www.ncbi.nlm.nih.gov/geo/), ArrayExpress (https://www.ebi.ac.uk/arrayexpress/), The Genotype-Tissue Expression (https://gtexportal.org/home/), The Cancer Genome Atlas (https://www.cancer.gov/about-nci/organization/ccg/research/structural-genomics/tcga), and
Sequence Read Archive (https://www.ncbi.nlm.nih.gov/sra). The accessions of datasets were listed in Additional file 1:Table S1.

\section{Competing interests}

The authors declare no competing interests.

\section{Author details}

${ }^{1}$ Department of Oncology, First Affiliated Hospital of Guangxi Medical University, No. 6 Shuangyong Rd, Guangxi Zhuang Autonomous Region, Nanning 530021, People's Republic of China. ' Department of Pathology, First Affiliated Hospital of Guangxi Medical University, No. 6 Shuangyong Rd, Guangxi Zhuang Autonomous Region, Nanning 530021, People's Republic of China. ${ }^{3}$ Department of Toxicology, College of Pharmacy, Guangxi Medical University, No. 22 Shuangyong Rd, Guangxi Zhuang Autonomous Region, Nanning 530021, People's Republic of China. ${ }^{4}$ Department of Pharmacy, Guangxi Medical University Cancer Hospital, No. 71 Hedi Rd, Guangxi Zhuang Autonomous Region, Nanning 530021, People's Republic of China. ${ }^{5}$ The Ultrasonics Division of Radiology Department, The First Affiliated Hospital of Guangxi Medical University, No. 6. Shuangyong Rd, Guangxi Zhuang Autonomous Region, Nanning 530021, People's Republic of China.

Received: 13 June 2021 Accepted: 30 July 2021

Published online: 21 August 2021

\section{References}

1. Zhao W, Ma B, Tian Z, Han H, Tang J, Dong B, et al. Inhibiting CBX4 efficiently protects hepatocellular carcinoma cells against sorafenib resistance. Br J Cancer. 2021;124:1237.

2. Fujita M, Yamaguchi R, Hasegawa T, Shimada S, Arihiro K, Hayashi S, et al. Classification of primary liver cancer with immunosuppression mechanisms and correlation with genomic alterations. EBioMedicine. 2020;53:102659.

3. Siegel RL, Miller KD, Fuchs HE, Jemal A. Cancer statistics, 2021. CA Cancer J Clin. 2021;71(1):7-33.

4. Zhang H, Dong P, Guo S, Tao C, Chen W, Zhao W, et al. Hypomethylation in HBV integration regions aids non-invasive surveillance to hepatocellular carcinoma by low-pass genome-wide bisulfite sequencing. BMC Med. 2020;18(1):200

5. Gu X, Guan J, Xu J, Zheng Q, Chen C, Yang Q, et al. Model based on five tumour immune microenvironment-related genes for predicting hepatocellular carcinoma immunotherapy outcomes. J Transl Med. 2021;19(1):26.

6. Liu S, Qiu J, He G, He W, Liu C, Cai D, et al. TRAlL promotes hepatocellular carcinoma apoptosis and inhibits proliferation and migration via interacting with IER3. Cancer Cell Int. 2021;21(1):63.

7. Razavi ZS, Asgarpour K, Mahjoubin-Tehran M, Rasouli S, Khan H, Shahrzad MK, et al. Angiogenesis-related non-coding RNAs and gastrointestinal cancer. Mol Ther Oncol. 2021;21:220-41.

8. Shafabakhsh R, Arianfar F, Vosough M, Mirzaei HR, Mahjoubin-Tehran M, Khanbabaei $\mathrm{H}$, et al. Autophagy and gastrointestinal cancers: the behind the scenes role of long non-coding RNAs in initiation, progression, and treatment resistance. Cancer Gene Ther. 2021.

9. de Martel C, Georges D, Bray F, Ferlay J, Clifford GM. Global burden of cancer attributable to infections in 2018: a worldwide incidence analysis. Lancet Glob Health. 2020;8(2):e180-90.

10. Tian MX, Liu WR, Wang H, Zhou YF, Jin L, Jiang XF, et al. Tissue-infiltrating lymphocytes signature predicts survival in patients with early/intermediate stage hepatocellular carcinoma. BMC Med. 2019;17(1):106.

11. Zhou KQ, Sun YF, Cheng JW, Du M, Ji Y, Wang PX, et al. Effect of surgical margin on recurrence based on preoperative circulating tumor cell status in hepatocellular carcinoma. EBioMedicine. 2020;62:103107.

12. Zou Y, Li H, Graham ET, Deik AA, Eaton JK, Wang W, et al. Cytochrome P450 oxidoreductase contributes to phospholipid peroxidation in ferroptosis. Nat Chem Biol. 2020;16(3):302-9.

13. Wang K, Wu Z, Si Y, Tang W, Xu X, Cheng Y, et al. Identification of LPCAT1 expression as a potential prognostic biomarker guiding treatment choice in acute myeloid leukemia. Oncol Lett. 2021;21(2):105. 
14. Jiang H, Li Z, Huan C, Jiang XC. Macrophage lysophosphatidylcholine acyltransferase 3 deficiency-mediated inflammation is not sufficient to induce atherosclerosis in a mouse model. Front Cardiovasc Med. 2018;5:192.

15. Wang B, Tontonoz P. Phospholipid remodeling in physiology and disease. Annu Rev Physiol. 2019;81:165-88.

16. Lebok P, von Hassel A, Meiners J, Hube-Magg C, Simon R, Höflmayer $D$, et al. Up-regulation of lysophosphatidylcholine acyltransferase 1 (LPCAT1) is linked to poor prognosis in breast cancer. Aging. 2019;11(18):7796-804.

17. Du Y, Wang Q, Zhang X, Wang X, Qin C, Sheng Z, et al. Lysophosphatidylcholine acyltransferase 1 upregulation and concomitant phospholipid alterations in clear cell renal cell carcinoma. J Exp Clin Cancer Res CR. 2017;36(1):66

18. Mansilla F, da Costa KA, Wang S, Kruhøffer M, Lewin TM, Orntoft TF, et al. Lysophosphatidylcholine acyltransferase 1 (LPCAT1) overexpression in human colorectal cancer. J Mol Med (Berl). 2009;87(1):85-97.

19. Wei C, Dong $X$, Lu H, Tong F, Chen L, Zhang R, et al. LPCAT1 promotes brain metastasis of lung adenocarcinoma by up-regulating PI3K/AKT/ MYC pathway. J Exp Clin Cancer Res CR. 2019;38(1):95.

20. Shida-Sakazume T, Endo-Sakamoto Y, Unozawa M, Fukumoto C, Shimada K, Kasamatsu A, et al. Lysophosphatidylcholine acyltransferase 1 overexpression promotes oral squamous cell carcinoma progression via enhanced biosynthesis of platelet-activating factor. PLOS ONE. 2015;10(3):e0120143.

21. Han C, Yu G, Mao Y, Song S, Li L, Zhou L, et al. LPCAT1 enhances castration resistant prostate cancer progression via increased mRNA synthesis and PAF production. PLoS ONE. 2020;15(11):e0240801.

22. Morita Y, Sakaguchi T, Ikegami K, Goto-Inoue N, Hayasaka T, Hang VT, et al. Lysophosphatidylcholine acyltransferase 1 altered phospholipid composition and regulated hepatoma progression. J Hepatol. 2013;59(2):292-9.

23. Bi J, Ichu TA, Zanca C, Yang H, Zhang W, Gu Y, et al. Oncogene amplification in growth factor signaling pathways renders cancers dependent on membrane lipid remodeling. Cell Metab. 2019;30(3):525-38.e8.

24. Han Y, Zhuang Q, Sun B, Lv W, Wang S, Xiao Q, et al. Crystal structure of steroid reductase SRD5A reveals conserved steroid reduction mechanism. Nat Commun. 2021;12(1):449.

25. Bidar M, Bahlakeh A, Mahmoudi M, Ahrari F, Shahmohammadi R, Jafarzadeh H. Does the application of GaAlAs laser and platelet-rich plasma induce cell proliferation and increase alkaline phosphatase activity in human dental pulp stem cells? Lasers in medical science. 2021.

26. Wu HY, Cai KT, Ma J, Chen G, Dang YW, Lu HP, et al. Evaluation of miR302b-5p expression and molecular mechanism in hepatocellular carcinoma: findings based on RT-qPCR and in silico analysis. Pathol Res Pract. 2019:215(7):152424

27. Xie Z, Dang Y, Wu H, He R, Ma J, Peng Z, et al. Effect of CELSR3 on the cell cycle and apoptosis of hepatocellular carcinoma cells. J Cancer. 2020;11(10):2830-44.

28. Gao L, Yan SB, Yang J, Kong JL, Shi K, Ma FC, et al. MiR-182-5p and its target HOXA9 in non-small cell lung cancer: a clinical and in-silico exploration with the combination of RT-qPCR, miRNA-seq and miRNA-chip. BMC Med Genomics. 2020;13(1):3.

29. Gao L, Xiong DD, He RQ, Yang X, Lai ZF, Liu LM, et al. MIR22HG as a tumor suppressive InCRNA in HCC: a comprehensive analysis integrating RTqPCR, mRNA-Seq, and microarrays. OncoTargets Ther. 2019;12:9827-48.

30. Itkonen HM, Urbanucci A, Martin SE, Khan A, Mathelier A, Thiede B, et al. High OGT activity is essential for MYC-driven proliferation of prostate cancer cells. Theranostics. 2019;9(8):2183-97.

31. Zhou C, Liu C, Liu W, Chen W, Yin Y, Li CW, et al. SLFN11 inhibits hepatocellular carcinoma tumorigenesis and metastasis by targeting RPS $4 \mathrm{X}$ via mTOR pathway. Theranostics. 2020;10(10):4627-43.

32. Nambiar DK, Aguilera T, Cao H, Kwok S, Kong C, Bloomstein J, et al. Galectin-1-driven T cell exclusion in the tumor endothelium promotes immunotherapy resistance. J Clin Invest. 2019;129(12):5553-67.
33. Shan D, Zou L, Liu X, Shen Y, Cai Y, Zhang J. Efficacy and safety of gabapentin and pregabalin in patients with vasomotor symptoms: a systematic review and meta-analysis. Am J Obstet Gynecol. 2020;222(6):564-79.e12.

34. Zhang L, Luo B, Dang YW, He RQ, Chen G, Peng ZG, et al. The clinical significance of endothelin receptor type B in hepatocellular carcinoma and its potential molecular mechanism. Exp Mol Pathol. 2019;107:141-57.

35. Hong W, Yuan H, Gu Y, Liu M, Ji Y, Huang Z, et al. Immune-related prognosis biomarkers associated with osteosarcoma microenvironment. Cancer Cell Int. 2020;20:83.

36. Bi F, Chen Y, Yang Q. Significance of tumor mutation burden combined with immune infiltrates in the progression and prognosis of ovarian cancer. Cancer Cell Int. 2020;20:373.

37. Kwon OS, Lee H, Kong HJ, Kwon EJ, Park JE, Lee W, et al. Connectivity map-based drug repositioning of bortezomib to reverse the metastatic effect of GALNT14 in lung cancer. Oncogene. 2020;39(23):4567-80.

38. Zhang $C$, Chen T, Li Z, Liu A, Xu Y, Gao Y, et al. Depiction of tumor stemlike features and underlying relationships with hazard immune infiltrations based on large prostate cancer cohorts. Briefings in bioinformatics. 2020

39. Wang Y, Kong W, Wang L, Zhang T, Huang B, Meng J, et al. Multiplepurpose connectivity map analysis reveals the benefits of esculetin to hyperuricemia and renal fibrosis. Int J Mol Sci. 2020;21(20):7695.

40. Liu Z, Wang X, Yang G, Zhong C, Zhang R, Ye J, et al. Construction of IncRNA-associated ceRNA networks to identify prognostic IncRNA biomarkers for glioblastoma. J Cell Biochem. 2020;121(7):3502-15.

41. Ye G, Qin Y, Wang S, Pan D, Xu S, Wu C, et al. Lamc1 promotes the Warburg effect in hepatocellular carcinoma cells by regulating PKM2 expression through AKT pathway. Cancer Biol Ther. 2019;20(5):711-9.

42. Li YL, Rao MJ, Zhang NY, Wu LW, Lin NM, Zhang C. BAY 87-2243 sensitizes hepatocellular carcinoma Hep3B cells to histone deacetylase inhibitors treatment via GSK-3 $\beta$ activation. Exp Ther Med. 2019;17(6):4547-53.

43. Ferrín G, Guerrero M, Amado V, Rodríguez-Perálvarez M, De la Mata M. Activation of mTOR signaling pathway in hepatocellular carcinoma. Int J Mol Sci. 2020:21(4):1266.

44. Li YL, Zhang NY, Hu X, Chen JL, Rao MJ, Wu LW, et al. Evodiamine induces apoptosis and promotes hepatocellular carcinoma cell death induced by vorinostat via downregulating HIF-1 a under hypoxia. Biochem Biophys Res Commun. 2018:498(3):481-6.

45. Miao JF, Peng YF, Chen S, Gao WJ, Yang QX, Zhu P, et al. A novel harmine derivative, $\mathrm{N}$-(4-(hydroxycarbamoyl)benzyl)-1-(4-methoxyphenyl)-9Hpyrido[3,4-b]indole-3-carboxamide (HBC), as histone deacetylase inhibitor: in vitro antiproliferation, apoptosis induction, cell cycle arrest, and antimetastatic effects. Eur J Pharmacol. 2018;824:78-88.

46. Berndt N, Eckstein J, Heucke N, Gajowski R, Stockmann M, Meierhofer D, et al. Characterization of lipid and lipid droplet metabolism in human HCC. Cells. 2019;8(5):512.

47. Sangineto M, Villani R, Cavallone F, Romano A, Loizzi D, Serviddio G. Lipid metabolism in development and progression of hepatocellular carcinoma. Cancers (Basel). 2020;12(6):1419.

48. Tian Y, Yang B, Qiu W, Hao Y, Zhang Z, Yang B, et al. ER-residential Nogo-B accelerates NAFLD-associated HCC mediated by metabolic reprogramming of oxLDL lipophagy. Nat Commun. 2019;10(1):3391.

49. Zhan T, Rindtorff N, Betge J, Ebert MP, Boutros M. CRISPR/Cas9 for cancer research and therapy. Semin Cancer Biol. 2019:55:106-19.

50. Liu F, Wu Y, Liu J, Ni RJ, Yang AG, Bian K, et al. A miR-205-LPCAT1 axis contributes to proliferation and progression in multiple cancers. Biochem Biophys Res Commun. 2020;527(2):474-80.

51. Zhao Y, Zhu C, Chang Q, Peng P, Yang J, Liu C, et al. MiR-424-5p regulates cell cycle and inhibits proliferation of hepatocellular carcinoma cells by targeting E2F7. PLoS ONE. 2020;15(11):e0242179.

52. Zhu M, Xu W, Wei C, Huang J, Xu J, Zhang Y, et al. CCL14 serves as a novel prognostic factor and tumor suppressor of HCC by modulating cell cycle and promoting apoptosis. Cell Death Dis. 2019;10(11):796.

53. Ying H, Ji L, Xu Z, Fan X, Tong Y, Liu H, et al. TRIM59 promotes tumor growth in hepatocellular carcinoma and regulates the cell cycle by degradation of protein phosphatase 1B. Cancer Lett. 2020;473:13-24. 
54. Tian L, Yao K, Liu K, Han B, Dong H, Zhao W, et al. PLK1/NF-KB feedforward circuit antagonizes the mono-ADP-ribosyltransferase activity of PARP10 and facilitates HCC progression. Oncogene. 2020;39(15):3145-62.

55. Xie B, Wang S, Jiang N, Li JJ. Cyclin B1/CDK1-regulated mitochondrial bioenergetics in cell cycle progression and tumor resistance. Cancer Lett. 2019:443:56-66

56. Willis $L$, Jönsson $H$, Huang KC. Limits and constraints on mechanisms of cell-cycle regulation imposed by cell size-homeostasis measurements. Cell Rep. 2020;32(6):107992.

57. Sun J, Zhu Z, Li W, Shen M, Cao C, Sun Q, et al. UBE2T-regulated H2AX monoubiquitination induces hepatocellular carcinoma radioresistance by facilitating CHK1 activation. J Exp Clin Cancer Res CR. 2020;39(1):222.

58. Jia M, Peng Z, Yang K, Su C, Wang Y, Yan C. A high-throughput targeted metabolomics method for the quantification of 104 non-polar metabolites in cholesterol, eicosanoid, and phospholipid metabolism: application in the study of a CCl(4)-induced liver injury mouse model. Analyst. 2020;145(10):3575-91

59. Sarvizadeh M, Hasanpour O, Naderi Ghale-Noie Z, Mollazadeh S, Rezaei $\mathrm{M}$, Pourghadamyari $\mathrm{H}$, et al. Allicin and digestive system cancers: from chemical structure to its therapeutic opportunities. Front Oncol. 2021;11:650256.

60. Zhang X, Shao J, Li X, Cui L, Tan Z. Docetaxel promotes cell apoptosis and decreases SOX2 expression in CD133-expressing hepatocellular carcinoma stem cells by suppressing the PI3K/AKT signaling pathway. Onco Rep. 2019;41(2):1067-74.

61. Tsilimigras DI, Ntanasis-Stathopoulos I, Moris D, Spartalis E, Pawlik TM. Histone deacetylase inhibitors in hepatocellular carcinoma: a therapeutic perspective. Surg Oncol. 2018;27(4):611-8.

62. Zhou L, Pan LC, Zheng YG, Du GS, Fu XQ, Zhu ZD, et al. Novel strategy of sirolimus plus thymalfasin and huaier granule on tumor recurrence of hepatocellular carcinoma beyond the UCSF criteria following liver transplantation: a single center experience. Oncol Lett. 2018;16(4):4407-17.

\section{Publisher's Note}

Springer Nature remains neutral with regard to jurisdictional claims in published maps and institutional affiliations.
Ready to submit your research? Choose BMC and benefit from:

- fast, convenient online submission

- thorough peer review by experienced researchers in your field

- rapid publication on acceptance

- support for research data, including large and complex data types

- gold Open Access which fosters wider collaboration and increased citations

- maximum visibility for your research: over $100 \mathrm{M}$ website views per year

At $\mathrm{BMC}$, research is always in progress.

Learn more biomedcentral.com/submissions 